\title{
GW23-e1473 INTERLEUKIN 33 MEDIATES THE INTESTINAL INFLUENCE ON THE HEART FUNCTION IN HEART FAILURE MICE-IDENTIFICATION OF GENES IN ILEUM RELATED TO HEART FAILURE USING GENE EXPRESSION PROFILING
}

doi:10.1136/heartjnl-2012-302920o.14

${ }^{1}$ Liu Haitao, ${ }^{2}$ Kitakaze Masafumi. ${ }^{1}$ Xijing Hospital, Fourth Military Medical University;

${ }^{2}$ Department of Cardiovascular Medicine, National Cerebral and Cardiovascular Center

Objectives Chronic heart failure (CHF) is a condition with a high morbidity and mortality, and accompanied by a range of concomitant disorders organs and systems. These disorders both contribute to the cause of the CHF and have a key role in the progression and response to treatment. However, less is known about the role of gastrointestinal tract in heart failure. We assumed that the changed endocrine function of gastrointestinal tract under heart failure condition might play some influence on the cardiac performance.

Methods To verify the assumption, we developed transverse aortic constriction (TAC) model and sham in mice. After 3 months, heart failure was confirmed by echocardiography. We analysed gene expression profiles of ileum and myocardial samples. Among all the genes detected on the microarray, we selected the genes whose expression was significantly different in ileum samples between sham and TAC groups. Subsequently, the functional analysis of these genes was performed and the biological functions most significant to the dataset were identified.

Results Based on the established statistical approach, 1467 genes were identified in ileum samples, of which 462 were up-regulated in TAC group. Further refining the screening condition and performing a literature-based search. Gene of interleukin 33 (II33) was identified with high expression level and strong connection with the regulation of cardiomyopathy.

Conclusions In conclusion, the identification of genes that are differentially expressed in ileum of heart failure mice supports the suggestion that microarray analyses may be useful in studying the role of gastrointestinal tract in heart failure. Inflammatory Il33 might mediate the intestinal influence on the heart function in heart failure mice. These results need further investigation and validation. 